\title{
Comparing Open-Circuit Voltage Hysteresis Models for Lithium-Iron-Phosphate Batteries
}

\author{
F. Baronti*, N. Femia ${ }^{\S}$, R. Saletti*, and W. Zamboni ${ }^{\S}$ \\ *Dipartimento di Ingegneria dell'Informazione, Università di Pisa, I-56122 Pisa, Italy \\ e-mail: f.baronti@iet.unipi.it \\ $\S$ Dipartimento di Ingegneria dell'Informazione, Ingegneria Elettrica e Matematica Applicata (DIEM) \\ Università degli Studi di Salerno, I-84084 Fisciano (SA), Italy \\ e-mailwzamboni@unisa.it
}

\begin{abstract}
The hysteresis in the state-of-charge (SoC) vs. open-circuit voltage characteristic of a lithium-iron-phosphate $\left(\mathrm{LiFePO}_{4}, \mathrm{LFP}\right)$ battery is modelled with two approaches. The first one is based on a first-order charge relaxation equation, the second one is the Preisach model implemented with the Everett function. The advantages and drawbacks of the methods are discussed. Simulation results are compared for a 20 A h LFP cell, stimulated with various $\mathrm{SoC}$ evolutions, allowing us to draw minor loops in the SoC-OCV plane. The results are also compared to experimental data.
\end{abstract}

\section{INTRODUCTION}

The design of the energy storage systems necessary in many applications of the industrial fields, such as electric transportation and utility grids, is becoming more and more attracted by the Lithium-ion (Li-ion) batteries [1]. Among the different variants of Li-ion batteries, lithium iron phosphate ( $\mathrm{LiFePO}_{4}$ or briefly LFP) is one of the most promising for Electric Vehicle (EV) or hybrid EV applications, offering high safety with a reasonable cost, lower than other lithiumbased batteries. LFP batteries are characterised by a flat OpenCircuit Voltage (OCV) when the State-of-Charge (SoC) ranges from $20 \%$ to $80 \%$. Moreover, OCV exhibits a pronounced hysteresis between the charge and discharge curves [2]-[3].

An accurate estimation of the battery SoC contributes to increase the safety and life expectancy of the battery, as well as to maximize the driving range of EVs and to reduce costs. SoC estimation is indeed a fundamental function in an advanced Battery Management System (BMS) [4]-[5]. SoC indicates the residual energy of the battery and it is usually expressed as a percentage of the battery capacity. Its knowledge allows one to keep the battery within the optimal operating conditions and to evaluate the remaining runtime of the overall system (e.g., the driving range of an EV). An accurate characterization of the hysteresis and affordable models of the phenomenon are required in order to obtain a precise estimation of the $\mathrm{SoC}$ and parameters for an LFP battery with a model-based observer, such as a filter [6]-[9].

Starting from the experimental data showing hysteresis in the SoC-OCV characteristic of a 20 A h LFP cell, two deeply different modelling approaches are compared in this paper. The first one is the so-called One-State Hysteresis (OSH) model, based on a fist-order relaxation equation. The battery

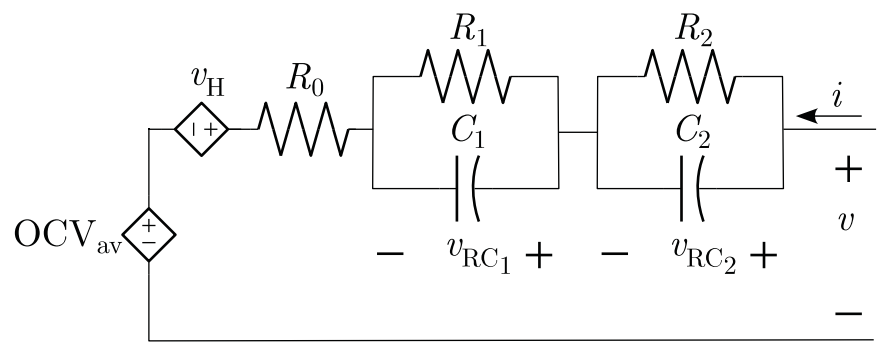

Fig. 1. Cell circuit output loop. The hysteretic voltage $v_{\mathrm{H}}$ depends on the SoC history.

static hysteretic response to a variation of $\mathrm{SoC}$ is contained in the hysteresis major loop and follows the forcing term in a "delayed" way (in fact, "delay" is the original meaning of the Greek-derived word "hysteresis") [3], [8]. The second model is the classical Preisach model, originally proposed in 1935 for magnetic materials [10] and then formalized as a fully-general mathematical tool in [11]. The Preisach model has been effectively proposed and used for NiMH batteries [12] and for Li-ion batteries [13]. The Preisach operator is implemented by means of the so-called Everett function, identified with a well-defined characterization procedure of the battery (first-order reversal (FOR) branches). The aim of this work is to draw a comparison of the two models.

\section{Modelling SoC - OCV Hysteresis}

\section{A. Equivalent Electric Circuit of a Li-ion Battery}

Let us consider a cell having a nominal capacitance $Q_{\mathrm{n}}$ and let us call $v$ the cell voltage and $i$ the cell current, with the passive sign convention. We also assume the cell temperature constant and equal to the room temperature $T$. The cell $\mathrm{SoC}$ is defined by a linear transformation of the charge $Q$ stored in the battery, such that $\operatorname{SoC}\left(Q=Q_{n}\right)=1$ and $\operatorname{SoC}(Q=0)=0$, where $Q_{n}$ is the normalisation battery capacity.

The equivalent electrical model of a Li-ion cell that includes the hysteresis voltage is shown in Fig. 1 [3]. The circuit is based on a model widely adopted in the literature [14] and it has been modified adding a voltage source accounting for hysteresis. The cell voltage is:

$$
v=\mathrm{OCV}_{\mathrm{av}}+v_{\mathrm{H}}+R_{0} i+v_{\mathrm{RC} 1}+v_{\mathrm{RC} 2},
$$


in which $\mathrm{OCV}_{\mathrm{av}}$ is the average OCV as a function of SoC, while $R_{0}, R_{k}$ and $C_{k}$ are the cell parameters $(k=1,2)$, and $v_{\mathrm{RC} k}$ is the solution of the following first-order equation:

$$
\frac{d v_{\mathrm{RC} k}}{d t}=-\frac{1}{R_{k} C_{k}} v_{\mathrm{RC} k}+\frac{i}{C_{k}} .
$$

It is worth noticing that the relaxation phenomena also occur when the battery current $i$ is equal to zero. In the following, a static analysis of hysteresis is carried out, so that we can safely assume $v_{\mathrm{RC} k}=0$.

Finally, the voltage source $v_{\mathrm{H}}$ in Fig. 1 represents the hysteretic voltage as a function of SoC, which is not necessarily a single-valued function.

\section{B. Hysteresis}

In order to model $v_{\mathrm{H}}$, two widely assessed methods are used: the first one is the so-called One-State Hysteresis (OSH) model [3], [8]. The hysteretic voltage is obtained by solving a first-order relaxation equation characterized by one relaxation parameter. The alternative approach adopted is the Preisach model [10], [13].

Before introducing the hysteresis models, we make the following assumptions: it is possible to define $v_{\mathrm{H}}$ as a function of the cell SoC, a function that depends on the SoC actual value and the SoC history. It is also possible to define a maximum hysteresis loop which "contains" any evolution and, thus, trajectory in the plane $\mathrm{SoC}-v_{\mathrm{H}}$ (state-space for hysteresis). The maximum hysteresis loop is defined by its upper bound $\mathrm{OCV}_{\mathrm{up}}(\mathrm{SoC})$ and its lower bound $\mathrm{OCV}_{\mathrm{lw}}(\mathrm{SoC})$. We also define the average open circuit voltage $\mathrm{OCV}_{\mathrm{av}}(\mathrm{SoC})$ as:

$$
\mathrm{OCV}_{\mathrm{av}}(\mathrm{SoC})=\frac{\mathrm{OCV}_{\mathrm{up}}(\mathrm{SoC})+\mathrm{OCV}_{\mathrm{lw}}(\mathrm{SoC})}{2} .
$$

The $\mathrm{OCV}_{\mathrm{av}}$ as defined in (3) is calculated from the experimental data and is plotted in Fig. 2. It is finally useful to define the non-negative function $E(\mathrm{SoC})$ representing the maximum hysteresis value to be added or subtracted to the average value $\mathrm{OCV}_{\mathrm{av}}$ :

$$
E(\mathrm{SoC})=\frac{\mathrm{OCV}_{\mathrm{up}}(\mathrm{SoC})-\mathrm{OCV}_{\mathrm{lw}}(\mathrm{SoC})}{2} .
$$

The function $E(\mathrm{SoC})$ is plotted in Fig. 3 .

\section{One-State Hysteresis Model}

Any SoC variation causes the hysteresis term $v_{\mathrm{H}}$ to vary with inertia. One way to represent the inertia in $\mathrm{SoC}$ is to use a first-order relaxation equation, as it happens for the voltage relaxation in an $\mathrm{RC}$ circuit. In this case we have to take into account that SoC variations might be negative, whereas time variations are positive in the time domain equations. With this idea in mind, we can write the differential equations describing the model in the $\mathrm{SoC}-v_{\mathrm{H}}$ plane when $d \mathrm{SoC} \geq 0$ :

$$
\frac{d v_{\mathrm{H}}}{d \mathrm{SoC}}=-\gamma\left[v_{\mathrm{H}}-E(\mathrm{SoC})\right] \quad \text { for } d \mathrm{SoC} \geq 0,
$$

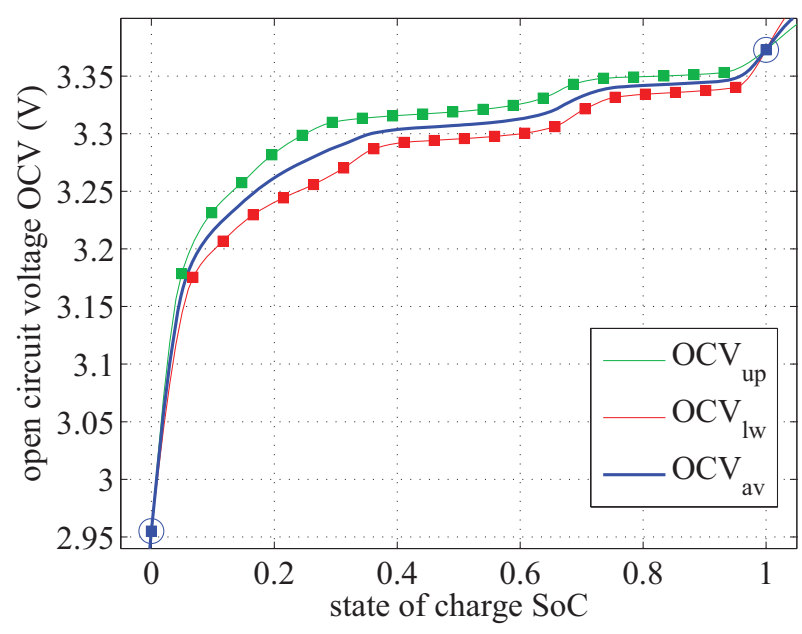

Fig. 2. Hysteresis major loop and average open circuit voltage defined by (3), as functions of SoC [3].

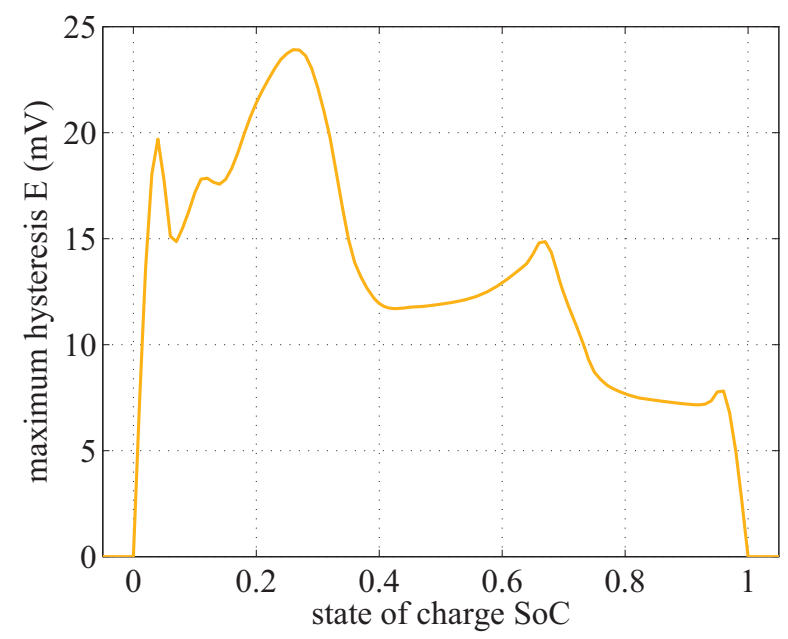

Fig. 3. Maximum positive hysteresis, defined according to (4) as a function of SoC [3].

being $\gamma>0$ and $v_{\mathrm{H}}=+E$ the steady-state solution. Conversely, when $d$ SoC $<0$, the steady-state solution is $v_{\mathrm{H}}=-E$, and the relaxation equation must be written as:

$$
\frac{d v_{\mathrm{H}}}{d \mathrm{SoC}}=\gamma\left[v_{\mathrm{H}}+E(\mathrm{SoC})\right] \quad \text { for } d \mathrm{SoC}<0 \text {. }
$$

In (5)-(6), $1 / \gamma$ is an adimensional constant (a sort of "stateof-charge constant") analogous to the time constant used in the time domain. Joining (5) and (6) we finally obtain:

$$
\frac{d v_{\mathrm{H}}}{d \mathrm{SoC}}=-\operatorname{sgn}(d \mathrm{SoC}) \gamma v_{\mathrm{H}}+\gamma E(\mathrm{SoC}) .
$$

In order to obtain a time domain equation from (7), we multiply both members by $\frac{d \mathrm{SoC}}{d t}$, obtaining:

$$
\frac{d v_{\mathrm{H}}}{d t}=-\operatorname{sgn}(d \mathrm{SoC}) \frac{d \mathrm{SoC}}{d t} \gamma v_{\mathrm{H}}+\gamma \frac{d \mathrm{SoC}}{d t} E(\mathrm{SoC})
$$


Considering that $i=Q_{\mathrm{n}} \frac{d \mathrm{SoC}}{d t}$, we have:

$$
\operatorname{sgn}(d \operatorname{SoC}) \frac{d \operatorname{SoC}}{d t}=\operatorname{sgn}(i) \frac{i}{Q_{\mathrm{n}}}=\frac{|i|}{Q_{\mathrm{n}}} .
$$

Substituting (9) into (8) we get:

$$
\frac{d v_{\mathrm{H}}}{d t}=-\frac{|i|}{Q_{\mathrm{n}}} \gamma v_{\mathrm{H}}+\operatorname{sgn}(i) \gamma \frac{|i|}{Q_{\mathrm{n}}} E(\operatorname{SoC})
$$

and, defining $\tau_{\mathrm{H}}=\frac{Q_{\mathrm{n}}}{\gamma|i|}$, we finally obtain:

$$
\frac{d v_{\mathrm{H}}}{d t}=-\frac{v_{\mathrm{H}}}{\tau_{\mathrm{H}}}+\operatorname{sgn}(i) \frac{E(\operatorname{SoC}(t))}{\tau_{\mathrm{H}}} .
$$

A circuit interpretation of (11) is given in [3].

\section{Preisach Hysteresis Model based on Everett Function}

The basic idea of the Preisach model is that the hysteretic response can be seen as the superposition of elemental relays' responses. The elemental relay $\gamma_{\alpha \beta}$ has two states: "up" $\left(\gamma_{\alpha \beta}=+1\right)$ and "down" $\left(\gamma_{\alpha \beta}=-1\right)$. Two switching thresholds allow the relay to change its state: $\mathrm{SoC}=\alpha$ is the switch-up threshold, while $\mathrm{SoC}=\beta$ is the "down" switching threshold. When SoC goes beyond $\alpha$, then the relay's response switches to +1 . Conversely, when SoC goes below $\beta$, its response turns to -1 . Between these thresholds, the state depends on the SoC history. If we write the superposition of the relays responses in the integral form, we obtain that the OCV can be expressed as a function of the independent variable $\operatorname{SoC}(t)$ as:

$$
\operatorname{OCV}(t)=\int_{\alpha \geq \beta} \mu(\alpha, \beta) \gamma_{\alpha \beta}\{\operatorname{SoC}(t)\} d \alpha d \beta,
$$

where the elemental relays are distributed according to the density function $\mu$, also called Preisach function.

Preisach theory is well assessed in the magnetic community. One of the main results of this theory prescribes that hysteresis must fulfill a necessary and sufficient condition in order to be represented by the so-called Preisach operator reported in (12). This condition consists of two properties: congruency and wiping out [11]. Such properties are extensively described in [11]. Here, we recall them very briefly.

The wiping out property refers to the circumstance that only the input "dominant" extrema are stored, while all the others are "wiped out" by the dominant ones. In other words, only a subset of the complete sequence of input extrema contributes to the state of the system. In fact, wiping out is also the most common mechanism for memory state updating and it can confidently be assumed as satisfied in the case of a battery. Conversely, the congruency property could only be assumed as an approximation in the battery case (and, more generally, in all practical cases of interest). Congruency means invariance of minor loops between the same input extrema if subjected to a vertical shift. The smaller the shift, the better the approximation. In [13] it is shown that congruency is better approximated when SoC is the input and OCV the output (and not vice versa), leading to write the superposition integral as in (12).
In order to identify the Preisach operator, one possibility is to identify the Preisach function $\mu$. We follow an alternative approach, using the so-called Everett function associated to the first-order reversal (FOR) hysteresis branches depicted in Fig. 4. The FOR branches are minor hysteresis loops that

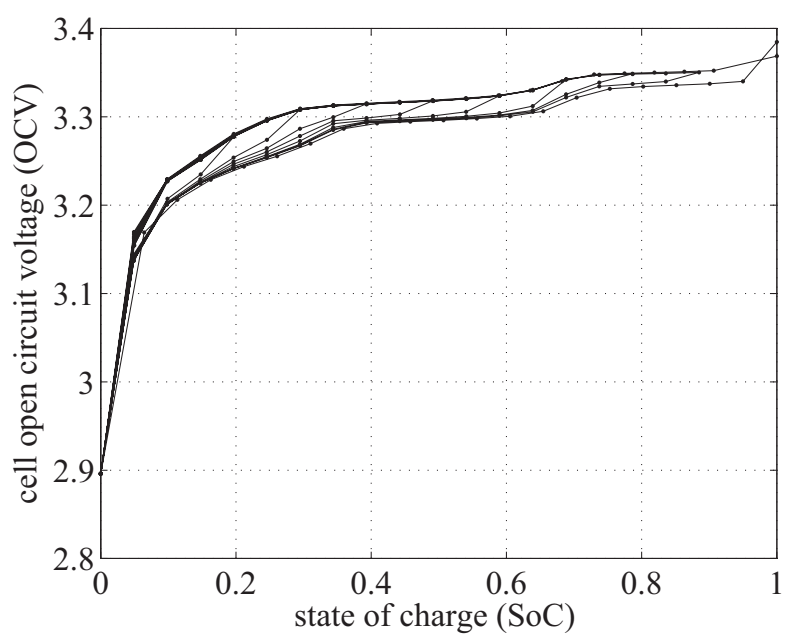

Fig. 4. First-order reversal (FOR) branches measured (in discharge) with a PCT characterization of a 20 A h LFP cell.

explore the hysteretic phenomenon with loops of decreasing amplitude from branch to branch. In our case, ten FOR branches are measured: they are uniformly-spaced with an increase of approximately $10 \%$ in SoC from branch to branch (from full charge state $\mathrm{SoC}=100 \%$ down to $10 \%$ ), with a SoC discretization step of $5 \%$. Temperature is kept constant at $298 \mathrm{~K}$ (more details on the experimental procedure will be given in the following Section). It is worth noting that the branches are very close to one another, but they do not overlap. Measuring FOR branches closer to each other would be desirable, but the experiment is highly time-consuming and would be unpractical. On the basis of the FOR branches, we define the so-called Everett function $\mathcal{E}$ as a function of the switching thresholds $\alpha$ and $\beta$, as it is done in [11]:

$$
\mathcal{E}(\alpha, \beta)=\frac{\mathrm{OCV}_{\alpha}-\mathrm{OCV}_{\alpha \beta}}{2},
$$

which is half of the output increment at point $\beta$ along a FOR branch starting in $\alpha$. The function could easily be rewritten in the following way:

$$
\mathcal{E}(\alpha, \beta)=\int_{\beta}^{\alpha} d \beta^{\prime} \int_{\beta^{\prime}}^{\alpha} \mu\left(\alpha^{\prime}, \beta^{\prime}\right) d \alpha^{\prime} .
$$

Geometrically, the value of the integral in (14) is associated to the upper-left vertex of the triangular domain of integration $T(\alpha, \beta)$.

The direct use of (14) would require the numerical evaluation of the integral as well as the determination of $\mu$ by deriving twice the experimental data. Geometrical considerations [11] prove that the integral (14) can be rewritten as a 
linear combination of the Everett function's values:

$$
\operatorname{OCV}(t)=2 \sum_{k}\left[\mathcal{E}\left(\alpha_{k}, \beta_{k-1}\right)-\mathcal{E}\left(\alpha_{k}, \beta_{k}\right)\right]-\mathcal{E}_{0}
$$

where all the appearing $\alpha_{i}$ and $\beta_{j}$ are the dominant extrema constituting the hysteresis state of the battery and $\mathcal{E}_{0}=\mathcal{E}(100 \%, 0 \%)$. The main benefit associated with the introduction of the Everett function is that the output is a linear combination of a set of Everett function values, which means a low computational burden.

\section{RESUlts}

\section{A. Experiments on a LFP Battery}

Pulsed Current Tests (PCT) have been performed on a LFP cell having a rated capacity of $20 \mathrm{~A} \mathrm{~h}$ and charge/discharge cutoff voltages of $3.65 \mathrm{~V}$ and $2.85 \mathrm{~V}$, in order to obtain the relationship between OCV and SoC [3], [15]. The cell's ambient temperature $T$ is kept constant at $298 \mathrm{~K}$ in a thermal chamber. Each PCT is preceded by an initialization procedure, including a complete discharge/charge cycle. Then, the battery is fed by a sequence of constant-current (CC) pulse steps (with duration $30 \mathrm{~min}$ ) determining a $5 \%$ variation of SoC, separated by rest steps (with duration $1 \mathrm{~h}$ ), in which the current is set to zero, so that the terminal voltage can relax toward its asymptotical value. The $\mathrm{CC}$ pulse value is $C / 10=2 \mathrm{~A}$ (one tenth of the rated capacity expressed in ampere-hours). As soon as the charge/discharge cutoff voltage is reached, charging/discharging proceeds in constant-voltage (CV) mode. These final conditions define the full-charge $(\mathrm{SoC}=100 \%)$ and full-discharge $(\mathrm{SoC}=0 \%)$ states and their corresponding OCV values, $\operatorname{OCV}(100 \%)$ and $\operatorname{OCV}(0 \%)$. A few tens of millivolts spreading of these values is observed among different experiments, so that $\operatorname{OCV}(0 \%)$ and $\operatorname{OCV}(100 \%)$ are defined by averaging the measured values.

TABLE I

SOC EVOLUTIONS

\begin{tabular}{cl}
\hline Label & SoC evolution (\%) \\
\hline \hline (a) & $100-0-100$ \\
(b) & $100-25-75-25-100$ \\
(c) & $100-40-60-40-100$ \\
\hline
\end{tabular}

Using the aforementioned procedure, three experimental PCTs, described in Table I, are carried out. In all the cases, the OCV experimental points are defined as the cell terminal voltage value reached at the end of each rest step [2], [3]. SoC value is determined by integrating current samples with the trapezoidal integration rule, and normalizing the charge obtained to the rated capacity. The current integral is reset in two points, when the battery reaches the full-charge and fulldischarge states as defined above. The SoC-OCV characteristic of the LFP cell is rather flat, with OCV variation less than $0.2 \mathrm{~V}$ in the SoC range from $10 \%$ to $90 \%$. Furthermore, the same kind of test has been used to obtain the FOR branches in Fig. 4, useful for the identification of the Preisach model.

\section{B. Simulation Results}

The two models described above have been used to reproduce the battery static OCV evolution, when the $\triangle$ SoC state of charge steps defined in the experiments are applied as a function of time. Three simulations are performed with the same charge variation profiles obtained with PCT described in Table I. The simulation initial condition is $\mathrm{SoC}=100 \%$, i.e., $Q_{n}=20 \mathrm{~A} \mathrm{~h}$.

The hysteresis parameter $\gamma$ in the OSH model is considered constant and it has been computed as a fitting parameter, by minimising the rms error between the simulation results and the experimental ones. The value that leads to the best achievable results is $\gamma=17.45$. Obviously, this procedure is very sensitive with respect to the data to be fitted, and the predictive capability of the method is thus limited in this case. On the contrary, Preisach model is identified on the basis of a very well defined training procedure, which ensures high repeatability and robustness.

Numerical results are reported in Fig. 5-Fig. 7. The values simulated with both OSH and Preisach models are plotted together with the experimental results. The first plot in each figure column shows the hysteretic behaviour in the SoC-OCV plane. The evolution of the OCV is represented as a "time simulation" (point-by-point OCV) in the second plot of each column. These plots allow us to distinguish the behaviour of branches that partially overlap. Finally, the last plot in each column shows the point-by-point error for both models. Table II collects the rms errors for OSH and Preisach model $\left(e_{\mathrm{rms}}^{\mathrm{OSH}}\right.$ and $e_{\mathrm{rms}}^{\mathrm{P}}$, respectively) and peak errors $\left(e_{\max }^{\mathrm{OSH}}\right.$ and $e_{\max }^{\mathrm{P}}$, respectively) in the three cases analyzed.

The results of the two methods are approximately equivalent in terms of rms error, as shown in Table II: they are always within a few percent. For the case (a), both OSH and Preisach models exhibit a pronounced local error in the full-discharge zone. The error is mainly due to the definition of OCV at $\mathrm{SoC}=0 \%$ reported above. However, this is not an issue because a large OCV variation corresponds to a very small SoC charge around full-discharge. Thus, the model prediction does not lead to large SoC estimation errors in this zone. Apart from the peak error at full-discharge, the errors with which both the models reproduce the hysteretic behaviour of the battery are satisfactorily low.

TABLE II

SIMULATION ERRORS NORMALIZED AT $\triangle \mathrm{OCV}=0.489 \mathrm{~V}$

\begin{tabular}{c|cc|cc} 
Case & $e_{\mathrm{rms}}^{\mathrm{OSH}}$ & $e_{\mathrm{rms}}^{\mathrm{P}}$ & $e_{\max }^{\mathrm{OSH}}$ & $e_{\max }^{\mathrm{P}}$ \\
\hline \hline (a) & $1.23 \%$ & $2.52 \%$ & $5.18 \%$ & $12.08 \%$ \\
(b) & $0.90 \%$ & $1.13 \%$ & $0.56 \%$ & $2.79 \%$ \\
(c) & $0.71 \%$ & $0.57 \%$ & $1.10 \%$ & $1.18 \%$ \\
\hline
\end{tabular}

\section{CONCLUSIONS}

The One State Hysteresis (OSH) and Preisach models have been used to describe the hysteresis in the state-of-charge vs. open circuit voltage characteristic of a lithium-iron-phospate 

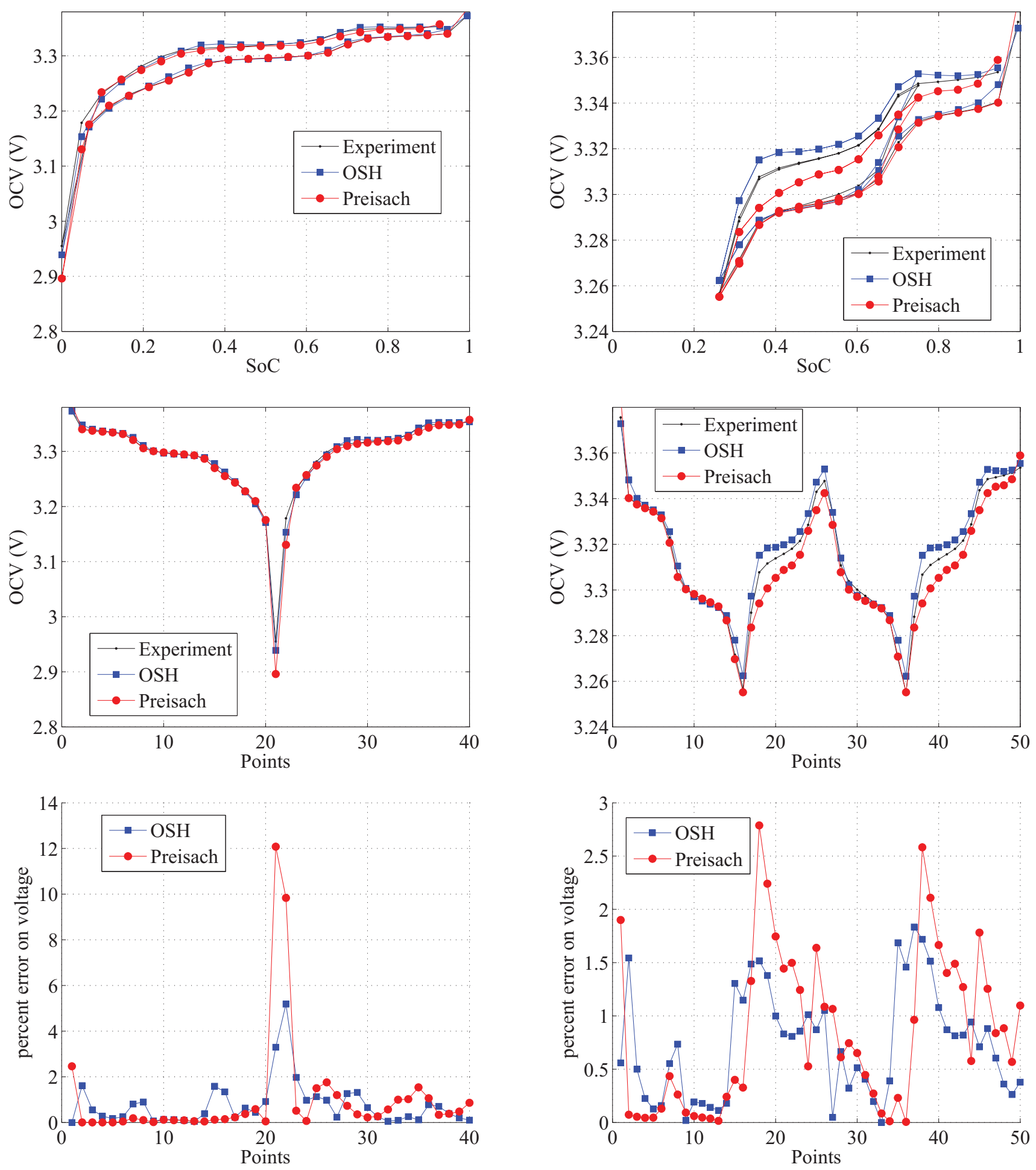

Fig. 5. Evolution (a): major loop. (Top) Response to SoC history in the SoC-OCV plane. (Middle) Point-by-point response to SoC history. (Bottom) Point-by-point error.

Fig. 6. Evolution (b): two nested minor loops. (Top) Response to SoC history in the SoC-OCV plane. (Middle) Point-by-point response to SoC history. (Bottom) Point-by-point error. 

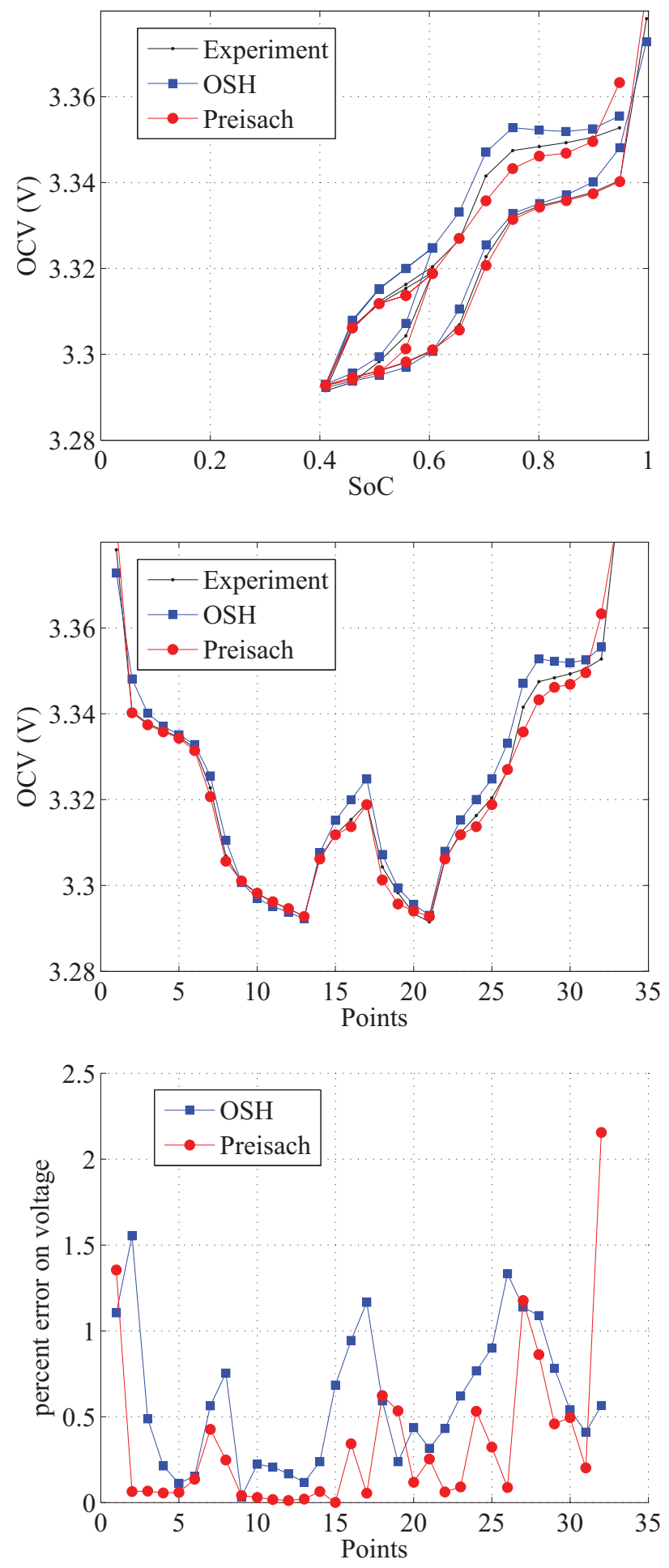

Fig. 7. Evolution (c): two nested minor loops. (Top) Response to SoC history in the SoC-OCV plane. (Middle) Point-by-point response to SoC history. (Bottom) Point-by-point error. battery. Both methods exhibit low rms errors with respect to experimental data in the three cases analyzed.

The OSH model is attractive for its simplicity and capability of capturing battery hysteretic effects with the use of a very simple relaxation equation and a single relaxation parameter. Nevertheless, the heuristic procedure used to find the relaxation parameter weakens this approach. On the other side, Preisach model implemented in terms of Everett function is characterized by a simple, well defined and reliable identification procedure based on the first-order reversal branches extraction. One of the most promising feature of the Preisach approach is the computationally affordable implementation that relies on rather simple operations (trapezoidal integration) on a well defined set of experimental data. The low computational complexity makes this last method very promising for an embedded implementation on low-cost BMS embedded platforms.

\section{REFERENCES}

[1] M. S. Whittingham, "History, Evolution, and Future Status of Energy Storage," Proc. IEEE, Vol. 100, pp. 1518-34, May 2012.

[2] M. A. Roscher, O. Bohlen, and J. Vetter, "OCV Hysteresis in LiIon Batteries including Two-Phase Transition Materials," International Journal of Electrochemistry, Vol. 2011, Article ID 984320, 2011.

[3] F. Baronti, W. Zamboni, N. Femia, R. Roncella, R. Saletti, "Experimental Analysis of Open-Circuit Voltage Hysteresis in Lithium-IronPhosphate Batteries," Proc. of IEEE IECON 2013, pp. 6726-31, 2013.

[4] H. Rahimi-Eichi, U. Ojha, F. Baronti, and M.-Y. Chow, "Battery Management System: An Overview of Its Application in the Smart Grid and Electric Vehicles," IEEE Ind. Electron. Mag., Vol. 7, No. 2, pp. 4-16, 2013.

[5] L. Lu, X. Han, J. Li, J. Hua, and M. Ouyang, , "A review on the key issues for lithium-ion battery management in electric vehicles," Journal of Power Sources, Vol. 226, pp. 272288, 2013.

[6] R. Restaino and W. Zamboni, "Comparing particle filter and extended kalman filter for battery State-Of-Charge estimation," Proc. of the IECON 2012 - 38th Annual Conference on IEEE Industrial Electronics Society, pp. 4018-4023, 2012.

[7] R. Restaino and W. Zamboni, "Rao-blackwellised particle filter for battery state-of-charge and parameters estimation," Proc. of the IECON 2013 - 39th Annual Conference on IEEE Industrial Electronics Society, pp. $6783-6788,2013$.

[8] G. L. Plett, "Extended Kalman filtering for battery management systems of LiPB-based HEV battery packs: Part 2. Modeling and identification," J. Power Sources, Vol. 134, No. 2, pp. 262-76, 2004.

[9] F. Baronti, W. Zamboni, N. Femia, R. Roncella, S. Rosi, S. Roberto, H. Rahimi Eichi, and M.-Y. Chow, "Parameter Identification of Li-Po batteries in electric vehicles: a comparative study," Proc. of the IEEE International Symposium on Industrial Electronics, pp. 1-7, 2013.

[10] F. Preisach, "Über die magnetische Nachwirkung," Zeitschrift für physik, Vol. 94, No. 5-6, pp. 277-302, 1935.

[11] I. D. Mayergoyz, Mathematical Models of Hysteresis, Springer, 1991.

[12] X. Tang, X. Zhang, B. Koch, D. Frisch, "Modeling and estimation of nickel metal hydride battery hysteresis for SOC estimation," Proc. of the IEEE International Conference on Prognostics and Health Management, 2008 (PHM 2008), pp. 1-12, 2008.

[13] F. Baronti, N. Femia, R. Saletti, C. Visone, W. Zamboni, "Hysteresis Modelling in Li-ion Batteries," IEEE Trans. Magn., do 10.1109/TMAG.2014.2323426, in press, 2014

[14] M. Chen and G. Rincon-Mora, "Accurate Electrical Battery Model Capable of Predicting Runtime and I-V Performance," IEEE Transactions on Energy Conversion, Vol. 21, pp. 504-511, 2006.

[15] A. Eddahech, O. Briat, and J.-M. Vinassa, "Thermal characterization of a high-power lithium-ion battery: Potentiometric and calorimetric measurement of entropy changes," Energy, Vol. 61, pp. 432-439, 2013. 